\title{
Calidad y visibilidad de las revistas científicas: el caso de PNA
}

\author{
Marta Molina*, Pedro Gómez*, María C. Cañadas*, Jesús Gallardo**, \\ José Luis Lupianez*
}

Resumen: La difusión internacional de la investigación de calidad constituye uno de los principales retos al que se enfrentan los consejos editoriales de las publicaciones científicas. Entre ellas se encuentra PNA, una revista española especializada en investigación en Educación Matemática. En este trabajo presentamos una reflexión sobre esta problemática desde nuestra experiencia como integrantes del consejo editorial de PNA. Con la descripción del proceso seguido para dar visibilidad internacional a la revista y garantizar su calidad científica, aspiramos aportar algunos referentes operativos de utilidad para aquellas iniciativas recientes que buscan difundir la producción investigadora de calidad.

Palabras clave: calidad, difusión, educación matemática, investigación, revistas científicas.

\section{Quality and visibility in scholarly journals: the case of PNA}

Abstract: The international dissemination of quality research constitutes one of the main challenges for editorial boards of scientific publications. Among these is PNA, a Spanish journal on mathematics education research. In this paper we present insights on this issue from our experience as members of the PNA editorial board. By describing the process followed for giving international visibility to the journal and for guaranteeing its scientific quality, we aim to provide some operative points of reference to be used in new projects intending to disseminate quality research production.

Keywords: quality, dissemination, mathematics education, research, scholarly journals.

\section{Introducción}

$P N A$ es la única revista especializada en investigación en Educación Matemática en España. Es una revista de acceso abierto, que publica estudios de investigación, experimentales o teóricos — tres artículos por número y cuatro números al año. Estos trabajos han sido revisados por pares de forma anónima, están es-

* Universidad de Granada. Correo-e: martamg@ugr.es, argeifontes@gmail.com, mconsu@ugr.es, lupi@ugr.es.

** Universidad de Málaga. Correo-e: gallardoromero@telefonica.net.

Recibido: 01-10-2010; 2. ${ }^{\mathrm{a}}$ versión: 24-01-2011; aceptado: 10-02-2011. 
critos en inglés o en español y siguen las normas de estilo APA. PNA es editada por el grupo de investigación FQM-193 «Didáctica de la Matemática: Pensamiento Numérico" (http://fqm193.ugr.es), del Plan Andaluz de Investigación, Desarrollo e Innovación de la Junta de Andalucía.

En este trabajo el consejo editorial de $P N A$ detalla los pasos que ha venido dando para dar visibilidad y calidad científica a la revista desde su lanzamiento, en septiembre de 2006, y los logros más significativos alcanzados. Este análisis pone de manifiesto la complejidad de los procesos involucrados y persigue ser de utilidad para investigadores de áreas afines implicados en iniciativas que buscan divulgar la actividad científica de calidad.

\section{Revistas de Investigación en Educación Matemática en España}

No todas las revistas de Educación Matemática poseen la misma relevancia como medio para la difusión de la producción investigadora. En términos genéricos, es posible distinguir entre: $a$ ) revistas especializadas que publican exclusivamente artículos de investigación sobre fenómenos ligados al aprendizaje y enseñanza de las matemáticas, y $b$ ) revistas divulgativas que fomentan el desarrollo de propuestas de innovación didáctica para el profesorado de matemáticas (Gallardo, 2006). En España, para presentar los resultados de sus investigaciones, los investigadores nacionales han utilizado fundamentalmente la vía ofrecida por Enseñanza de las Ciencias, revista de investigación de las del primer tipo aunque no es específica de Educación Matemática. De manera simultánea, buscando dar divulgación a los trabajos en las comunidades de profesores de matemáticas, también ha sido tradicional el uso de revistas del segundo tipo, como son Suma, Épsilon o Números.

Resultaba sorprendente que, más de 20 años después de que la Didáctica de la Matemática ${ }^{1}$ se constituyera como disciplina académica en España, con la creación de departamentos universitarios en el área y el ofrecimiento de programas de doctorado (Rico y otros, 2002), no existiese ninguna revista española especializada en la investigación en esta disciplina. Esta situación contrasta, sin duda, con la de países vecinos como Portugal o Francia donde desde hace años se viene publicando la investigación propia en revistas especializadas en esta área como Quadrante (1992) o Recherches en Didactique des Mathématiques (1980), respectivamente. También en México, el Comité Latinoamericano de Matemática Educativa creó en 1998 la Revista Latinoamericana de Investigación en Matemática Educativa (Relime). Durante ocho años, hasta la aparición de PNA, ésta fue la única revista especializada en investigación en Educación Matemática publicada en un país de habla hispana.

${ }^{1}$ A nivel internacional a esta disciplina y a su área de investigación asociada se le denomina Educación Matemática.

Rev. Esp. Doc. Cient., 34, 2, abril-junio, 266-275, 2011. ISSN: 0210-0614. doi:10.3989/redc.2011.2.802 
Se puede conjeturar que la carencia en España de revistas de investigación en Educación Matemática es consecuencia del carácter emergente que esta área de investigación tiene en España y, posiblemente, de la poca presión que, durante varios años, existió en las universidades españolas para la publicación de trabajos de investigación en revistas prestigiosas. La falta de revistas específicas de esta área con reconocimiento internacional, unida al esfuerzo adicional de escribir en otro idioma para publicar en una revista extranjera de calidad, ha hecho que sean pocos los investigadores que cuentan con publicaciones de impacto internacional. Lo cierto es que entre el 2000 y el 2008, los investigadores españoles publicaron solamente cinco artículos en revistas clasificadas en el Journal Citation Report-Social Sciences (Llinares, 2008, p. 29).

La escasa visibilidad de los investigadores españoles en el ámbito internacional, expresada en términos de artículos, contrasta con la elevada participación que los investigadores nacionales vienen teniendo en diversos congresos y reuniones relevantes, nacionales e internacionales, como son los simposios de la Sociedad Española de Investigación en Educación Matemática (SEIEM) y las conferencias anuales del International Group for the Psychology of Mathematics Education (PME) ${ }^{2}$. En la actualidad, las actas de estas conferencias constituyen un referente básico y representativo para constatar los rasgos distintivos de la investigación que se realiza en Educación Matemática a nivel nacional e internacional, respectivamente.

En definitiva, hasta ahora no han sido muchos los investigadores españoles que, habiendo realizado estudios de calidad y publicado sus resultados en actas de reuniones científicas prestigiosas, hayan publicado estos resultados en revistas incluidas en índices de impacto influyentes. No obstante, sospechamos que esta situación podría cambiar a raíz de los recientes requisitos de acreditación y habilitación en la universidad española, que han generado un interés y una necesidad crecientes de los investigadores de publicar en revistas indexadas.

En todo caso, reconocemos que la Educación Matemática a nivel internacional sigue siendo una disciplina joven en comparación con otras muchas. Ésta puede ser también una de las razones por la que en la actualidad sólo tres revistas específicas de investigación estén incluidas en el Social Science Citation Index (SSCI): Journal for Research in Mathematics Education (Estados Unidos), Revista Latinoamericana de Investigación en Matemática Educativa (México) y Boletim de Educação Matemática (Brasil).

\section{Creación y lanzamiento de PNA}

En el contexto anteriormente descrito, PNA se creó con la intención de ser un medio de difusión de calidad de trabajos de investigación en Educación Matemática, buscando mejorar la situación mencionada y tratando de dar respuesta

2 Las actas del PME aparecen indexadas en el Conference Proceedings Citation Index-Social Science \& Humanities (CPCI-SSH). 
a la ausencia de revistas españolas especializadas en investigación en esta área. El lanzamiento de la revista tuvo lugar con un esquema inicial en el que la mayoría de los artículos publicados fueron por invitación y uno de los objetivos era proporcionar visibilidad a trabajos de calidad previamente presentados en congresos nacionales e internacionales.

$P N A$ sale a la luz en Septiembre de 2006 por la iniciativa de cuatro estudiantes de doctorado en Didáctica de la Matemática de la Universidad de Granada ${ }^{3}$ y con el apoyo del grupo de investigación FQM-193, integrado por 25 investigadores de universidades andaluzas, que proporciona el soporte institucional y financiero necesario. El respaldo internacional, por su parte, viene dado por el comité científico de la revista que está constituido, desde sus inicios, por 11 investigadores de prestigio en el área, de 10 países diferentes, entre ellos Brasil, Canadá, Dinamarca, Estados Unidos, Francia, México y Portugal. Hasta la fecha se han publicado los cinco primeros volúmenes de la revista, con cuatro números cada uno publicados en los meses de septiembre, enero, marzo y junio.

\section{Difusión de PNA}

Desde la creación de $P N A$, el consejo editorial ha aplicado variadas estrategias dirigidas a dar difusión a la revista a nivel nacional e internacional. Con esta intención la revista es bilingüe (inglés o español), publica con regularidad al menos un artículo en inglés y existe en dos formatos, uno impreso y otro electrónico (http://www.pna.es).

\subsection{Versión impresa}

El propósito de la versión impresa de $P N A$ es dar a conocer gratuitamente la revista, de una forma directa y cercana, a un número reducido de investigadores e instituciones reconocidos en el área a nivel internacional. Para ello, se configuró una lista de destinatarios de interés a los que enviar la revista en formato impreso. Dicho listado estaba inicialmente formado por:

- Bibliotecas y departamentos de las universidades españolas donde se imparte docencia en el área de Didáctica de la Matemática.

- Editores de revistas nacionales e internacionales que publican trabajos de Educación Matemática incluidas en el SSCI o en IN-RECS, y algunas otras que destacan por su visibilidad o relevancia en el área.

3 En cierta forma, la aparición de $P N A$ en la escena científica puede entenderse como un resultado visible derivado de la consolidación de los programas de doctorado y del avance de la formación de investigadores en Didáctica de la Matemática en España. En la actualidad, todos los miembros del consejo editorial de la revista son doctores en el área.

Rev. Esp. Doc. Cient., 34, 2, abril-junio, 266-275, 2011. ISSN: 0210-0614. doi:10.3989/redc.2011.2.802 
- Coordinadores de la Sociedad Española de Investigación en Educación Matemática (SEIEM).

- Centros de documentación, bases de datos e índices de áreas afines.

- Investigadores internacionales en Educación Matemática que son colaboradores habituales del comité editorial u otros miembros del grupo editor de la revista.

Siguiendo este procedimiento, en la actualidad, la versión impresa de PNA se envía a 184 destinatarios nacionales e internacionales de América, Asia, Europa y Oceanía. Con los destinatarios de la revista se ha seguido un proceso de selección estricto para lograr la mayor difusión de la revista, sin que eso suponga un incremento notable de los costos.

\subsection{Versión electrónica}

Paralelamente, se ha potenciado la divulgación de la revista en su formato electrónico con diversas acciones entre las que destacamos la creación de un servicio de suscripción electrónica, que permite a los lectores recibir un aviso con la publicación de cada número, y la inclusión de enlaces a la versión digital de la revista en varias páginas relacionadas con la Educación Matemática. En la actualidad hay 340 personas suscritas electrónicamente y son más de 1.300 las páginas web que incluyen enlaces a $P N A$. Asimismo, se ha dado publicidad a la revista en congresos nacionales e internacionales de investigadores en Educación Matemática.

El esfuerzo de promoción de ambas versiones ha tenido como resultado destacable que la página web de la revista aparezca como primera opción en Google cuando se hacen búsquedas por la frase «investigación en didáctica de la matemática" y octava con la búsqueda "didáctica de la matemática" ${ }^{4}$.

\section{Visibilidad de PNA en fuentes de información secundarias}

Además de la difusión impresa y electrónica de la revista, también se ha ido ampliando progresivamente el listado de índices, bases de datos científico-técnicas, directorios de revistas, catálogos de bibliotecas, motores de búsqueda académicos y repositorios, en los que está incluida $P N A$. La solicitud de inclusión en estas fuentes de información secundarias es una actividad constante del consejo editorial.

Este proceso es complejo debido a la ingente cantidad existente de este tipo de fuentes y la diversidad de características y modos de gestión de las mismas. En primer lugar, ha sido necesario identificar fuentes de interés susceptibles de

\footnotetext{
${ }^{4}$ Resultados correspondientes al día 10 de agosto de 2010.
} 
incluir a PNA e indagar en el proceso de selección de publicaciones que emplean. En este primer paso encontramos dificultades para discernir entre fuentes secundarias, al no ser clara la jerarquía entre las mismas, así como para identificar las posibilidades reales de inclusión en cada fuente a fin de optimizar los esfuerzos. A falta de otros criterios, nos hemos decantado por aquellas fuentes que incluyen a revistas del área o de áreas afines, así como aquellas que hemos ido conociendo a lo largo de nuestra actividad investigadora.

Simultáneamente, identificados los criterios de calidad exigidos por las fuentes seleccionadas y buscando su cumplimiento, hemos ido definiendo el formato actual de la revista y modificando su política editorial. Hemos pasado de publicar trabajos solicitados por invitación, a abrir la recepción de manuscritos a toda la comunidad internacional de Educación Matemática. En cuanto al formato, hemos ido incluyendo progresivamente los elementos exigidos por las fuentes de información seleccionadas. En particular hemos adaptado el formato de la revista a los criterios Latindex ${ }^{5}$ que son bastante exhaustivos y engloban la mayoría de los estándares de publicación de revistas científicas exigidos internacionalmente. La familiarización con estos criterios ha permitido definir un formato estable para la revista, susceptible únicamente de mejoras en la disposición o apariencia de sus diferentes componentes.

En relación con la política editorial, los criterios que debe satisfacer una revista para ser reconocida por la comunidad científica, no son tan fácilmente identificables y "en algunos casos" no son ni siquiera accesibles para una revista en sus primeros años de existencia. Entre estos criterios cabe destacar la aplicación obligatoria y rigurosa del proceso de revisión por pares, la inclusión de trabajos de autores de variada procedencia a nivel internacional, la citación de revistas de relevancia internacional, la transparencia del proceso editorial desde la recepción de los manuscritos hasta su publicación, y la inclusión de citas de la propia revista en otras publicaciones relevantes a nivel internacional (Ruiz-Pérez y otros, 2006).

Fruto del trabajo realizado y guiados por el cumplimiento de estos criterios, en la actualidad $P N A$ se encuentra indexada en 26 fuentes de información secundaria nacionales e internacionales. En la tabla I presentamos las fuentes ${ }^{6}$ en las que se encuentra $P N A$ en relación con las de las dos revistas en español más relevantes ${ }^{7}$ dentro del área de las Ciencias Sociales que incluyen trabajos de Educación Matemática.

Si bien la presencia de $P N A$ en fuentes secundarias es destacada, su corto período de existencia es un factor limitante para la inclusión en algunas fuentes relevantes, así como para su evaluación mediante indicadores basados en el número de citas que reciben sus artículos, tales como el índice de impacto de IN-RECS.

\footnotetext{
5 Véase http://www.latindex.org

${ }^{6}$ Presentamos conjuntamente diferentes tipos de fuentes secundarias sin distinguir si se trata de bases de datos, repositorios, índices, buscadores o portales de revista de asociaciones y organizaciones científicas.

${ }^{7}$ Las hemos considerado más relevantes por estar incluidas en la Web of Science.
} 


\section{TABLA I}

Comparativa entre las fuentes de información secundarias en las que están incluidas PNA, Enseñanza de las Ciencias y Relime

\begin{tabular}{|c|c|c|c|}
\hline & \multicolumn{3}{|c|}{ Revista } \\
\hline & $P N A$ & $\begin{array}{c}\text { Enseñanza } \\
\text { de las Ciencias }\end{array}$ & Relime \\
\hline Catálogo Latindex & $\checkmark$ & $\checkmark$ & $\checkmark$ \\
\hline INRECS & $\checkmark$ & $\checkmark$ & \\
\hline SSCI-ISI Web of Knowledge & & $\checkmark$ & $\checkmark$ \\
\hline IRESIE & $\checkmark$ & & $\checkmark$ \\
\hline ISOC-CSIC-Revistas de Ciencias Sociales y Humanidades & $\checkmark$ & $\checkmark$ & \\
\hline Dialnet & $\checkmark$ & & $\checkmark$ \\
\hline Biblioteca digital (OEI) & $\checkmark$ & $\checkmark$ & \\
\hline Academic search complete (EBSCO) & $\checkmark$ & & $\checkmark$ \\
\hline PSYHLIT & & $\checkmark$ & \\
\hline Informe académico (GALE) & $\checkmark$ & & $\checkmark$ \\
\hline CLASE, Scielo, IBZ & & & $\checkmark$ \\
\hline $\begin{array}{l}\text { Biblioteca Digital de Matemáticas, BIMPE, CBNE, DICE, } \\
\text { DIGIBUG, Directory of Open Access Scholarly Journals in } \\
\text { Education, DOAJ, e-revist@s, EDNA, Funes, Genamics Jo- } \\
\text { urnalSeek, Google Académico, ICAAP, Informe Académico, } \\
\text { MathEduc, OASISBR, REBIUN, ScientificCommons, ZDB }\end{array}$ & $\checkmark$ & & \\
\hline
\end{tabular}

Estos índices, que se calculan con base en una combinación del número de citaciones y número de artículos publicados en una revista, son actualmente los primeros criterios considerados en la evaluación de la investigación en España (Buela-Casal, 2010) y de la difusión de una revista a nivel internacional.

\section{Calidad de los trabajos publicados en PNA}

Junto con la difusión, la calidad de los artículos publicados es otra de las principales cuestiones que preocupa a los editores de las revistas científicas españolas (Abadal y Rius, 2008) y, en particular, al consejo editorial de $P N A$ desde el lanzamiento de la misma. Si bien ambos objetivos pueden abordarse de forma independiente, en realidad están muy relacionados. A mayor calidad, más posibilidades de difusión; e inversamente, cuanto mayor es la difusión de una revista, más atractiva resulta para los autores del área y mayor puede ser la calidad de los trabajos que se publiquen en ella. Muestra de la interrelación entre calidad y difusión es el hecho de que las agencias de evaluación de la actividad inves- 
tigadora en España consideran como indicios de la calidad de las publicaciones la difusión que tenga el medio en el que aparezca dicha publicación.

En Educación Matemática permanece abierto el debate sobre los requisitos que debieran cumplir las investigaciones para poder ser consideradas de calidad. De acuerdo con lo subrayado por algunos de los autores que han venido reflexionando sobre esta cuestión en los últimos años (Romberg, 1992; Sierpinska y otros, 1993), todo estudio sobre cualquier cuestión de interés para la Educación Matemática debería manifestar suficientes garantías de validez, racionalidad, originalidad, rigurosidad, reproductibilidad o relevancia, entre otros criterios. Pero además de cumplir con estos criterios, que caracterizan a la calidad en un sentido clásico, la investigación también habría de garantizar su calidad en el sentido más amplio subrayado por Simon (2004), sobre todo en lo concerniente a proporcionar conocimiento que permita el avance del área de forma significativa.

En el ámbito de las publicaciones periódicas en el área, también son recurrentes las reflexiones en torno al procedimiento y los criterios de calidad empleados por los comités editoriales de las principales publicaciones para la aceptación de un trabajo para su publicación (Hanna, 1998).

Con estas premisas y referentes, $P N A$ persigue desde su inicio aumentar su calidad como publicación científica. Puesto que resulta difícil concretar en unas instrucciones breves y concisas una posición sobre lo que constituye un manuscrito de investigación de calidad e impacto, una de las dificultades que se presentan en este proceso es la selección de unos criterios válidos y eficaces que guíen la revisión de los manuscritos. Las acciones del consejo editorial en este sentido han consistido hasta el momento en realizar una cuidada selección de los revisores, entre los expertos del área, a los que se les solicita una atenta revisión de los manuscritos que busque incrementar o, al menos mantener, la calidad de los trabajos que se publican en la revista.

En la actualidad, PNA presenta unos índices cienciométricos muy positivos para su antigüedad (Bracho, 2010):

- Su índice de colaboración es de 2,26, superior al número de dos firmas por artículo que usualmente se establece para las Ciencias Sociales en España.

- Todos los artículos han presentado citas bibliográficas y se constata una media elevada de citas a artículos y tesis doctorales.

- Las citas, que en su mayoría son en inglés, tienen una antigüedad media de 9,22 años, que es inferior a la media en Ciencias Humanas y Sociales en España.

Por otra parte, creemos que el número elevado de fuentes de información secundarias en las que se encuentra $P N A$ y el esfuerzo por mejorar la calidad de su contenido están en el origen de un aumento reciente en el número de manuscritos que recibimos para su publicación.

Con el objetivo de aumentar la visibilidad de la revista vemos necesario promover la citación regular de la misma en otras publicaciones, principalmente de 
impacto. Para ello, el consejo editorial busca incrementar la calidad de los trabajos que se publican en $P N A$, así como desarrollar sistematicidad en la aplicación de criterios de calidad para la eventual publicación de los artículos recibidos. En todo caso, el déficit de publicaciones influyentes en Educación Matemática junto con la juventud de la revista, dificulta en estos momentos el alcance de nuestras pretensiones de difusión y calidad pues hace que los científicos de alto nivel del área todavía no consideren a $P N A$ como vía preferente de difusión de sus resultados.

\section{Consideraciones finales}

La creación de PNA tuvo lugar para dar respuesta a la necesidad de disponer de una publicación seriada de calidad en el área de Educación Matemática en España en la que sus investigadores pudiesen compartir, difundir y someter a la valoración de la comunidad científica los trabajos que vienen realizando. Con este propósito primordial iniciamos una labor sistemática y compleja, que se ha visto condicionada con los referentes y requerimientos propios de la literatura de investigación y de la juventud del área. Partiendo de la publicación por invitación de algunos de los trabajos de calidad de determinados investigadores del área, $P N A$ se ha abierto posteriormente a toda la comunidad internacional de Educación Matemática con objeto de constituirse, a largo plazo, como un referente en las revistas de investigación de impacto y relevancia en esta área.

En el proceso de difusión de $P N A$, hemos seguido en gran medida las líneas básicas de actuación para mejorar la difusión e impacto de las revistas científicas que señalan Abadal y Rius (2008): digitalización de contenidos, inclusión en portales, difusión en libre acceso, elaboración de versiones multilingües, comunicación de novedades, medición de la audiencia, e inclusión en bases de datos. Si bien podrían realizarse más acciones en alguna de estas líneas, algunas no se han realizado por resultar muy costosas - e.g., publicar todos los artículos de la revista en varios idiomas.

Las diversas estrategias mencionadas buscan dar visibilidad directa a la revista a través de sus suscriptores y de su presencia en bibliotecas, así como visibilidad indirecta por medio de su presencia en fuentes secundarias de información (Román y otros, 2001).

Alcanzado un formato estable de la revista, que cumple los estándares de publicación exigidos internacionalmente, nuestros esfuerzos se centrarán, en la medida de lo posible, en dos acciones que se retroalimentan en la búsqueda de visibilidad y calidad: incrementar el número y relevancia de las fuentes en las que está incluida la revista y desarrollar sistematicidad en la aplicación de criterios de calidad para la selección de manuscritos.

Como hemos señalado, la consecución de todos estos objetivos implica un esfuerzo sistemático y constante del consejo editorial para alcanzar los requerimientos de la comunidad científica internacional para las publicaciones especializadas. 


\section{Bibliografía}

Abadal, E., y Rius, L. (2008). Revistas científicas de las universidades españolas: acciones básicas para aumentar su difusión e impacto. Revista Española de Documentación Científica, vol. 31 (2), 242-262,

Bracho, R. (2010). Visualización de la investigación en Educación Matemática en España. Análisis bibliométrico y conceptual de la producción de artículos científicos (2003-2008). Tesis doctoral no publicada, Universidad de Córdoba.

Buela-Casal, G. (2010). Scientific journal impact indexes and indicators for measuring researchers' performance. Revista de Psicodidáctica, vol. 15 (1), 3-19.

Gallardo, J. (2006). Aportes a la investigación en educación matemática en contextos latinoamericanos desfavorables: el acceso a la información a texto completo. UNIÓN Revista Iberoamericana de Educación Matemática, 6, 31-43.

Hanna, G. (1998). Evaluating research papers in mathematics education. En: A. Sierpinska y J. Kilpatrick (editores), Mathematics education as a research domain: A search for identity. Dordrecht: Kluwer Academic Publishers.

Llinares, S. (2008). Agendas de investigación en Educación Matemática en España. Una aproximación desde ISI-Web of Knowledge y ERIH. En: R. Luengo; B. Gómez, M. Camacho y L. Blanco (editores), Actas del XII Simposio de la Sociedad Española de Investigación en Educación Matemática, pp. 25-54. Badajoz: SEIEM.

Rico, L.; Castro, E., y Sierra, M. (2002). El área de conocimiento de "Didáctica de la Matemática". Revista de Educación, 328, 35-58.

Román, A. (coord.); Giménez, E.; Gómez, I.; Martín-Sempere, M. J.; Páez, J.; Urdín, C., y Vázquez, M. (2001). La edición de revistas científicas: guía de buenos usos. Madrid: CINDOC.

Romberg, T. A. (1992). Perspectives on scholarship and research methods. En: D. A. Grouws (ed.), Handbook of research on mathematics teaching and learning. New York: MacMillan Publishing Company.

Ruiz-Pérez, R.; Delgado, E., y Jiménez-Contreras, E. (2006). Criterios del Institute for Scientific Information para la selección de revistas científicas. Su aplicación a las revistas españolas: metodología e indicadores. International Journal of Clinical and Health Psychology, vol. 6 (2), 401-424.

Simon, M. A. (2004). Raising issues of quality in Mathematics Education research. Journal for Research in mathematics education, vol. 35 (3), 157-163.

Sierpinska, A.; Kilpatrick, J.; Balacheff, N.; Howson, A. G.; Sfard, A., y Steinbring, H. (1993). What is research in Mathematics Education, and what are its results? Journal for Research in mathematics education, vol. 24 (3), 274-278. 